\title{
Targeted Therapies in HER2-Positive Breast Cancer - a Systematic Review
}

\author{
Amelie Schramm Nikolaus De Gregorio Peter Widschwendter Visnja Fink Jens Huober \\ Department of Gynecology and Obstetrics, University Hospital UIm, Germany
}

\author{
Keywords \\ HER2-positive breast cancer - Targeted therapies . \\ Dual HER2 blockade
}

\begin{abstract}
Summary
About $20 \%$ of all breast cancer patients have a human epidermal growth factor receptor 2 (HER2)-positive breast tumor. This entity underwent an impressive change in prognosis, with notable improvement of progression-free survival and overall survival. Due to more aggressive tumors and no specific therapy, HER2 overexpression was historically seen as a negative prognostic marker, with worse prognosis and increased risk of recurrent disease. Trastuzumab, the first anti-HER2 antibody, revolutionized the systemic therapy options in HER2-positive breast cancer and initiated several targeted therapies and more personalized treatment strategies. Over the years, multiple HER2-targeting drugs stepped into clinical practice, for the curative as well as the metastatic situation. This review summarizes the targeted treatment options in HER2-positive breast cancer and their current impact in the clinical routine. Results of the most outstanding trials in HER2-targeted therapies and important ongoing trials are subsequently described for an up-to-date overview.
\end{abstract}

\section{Introduction}

Worldwide, breast cancer is the most frequent cancer in women and, thus, disease management is well investigated. Nevertheless, due to various histological subtypes with mainly ductal and lobular adenocarcinomas and differences in receptor expression and ge- netic profiles, multiple therapy options exist. The majority of the adenocarcinomas are hormone receptor (HR) positive and approximately $20 \%$ of the malignant breast tumors show an overexpression of human epidermal growth factor receptor 2 (HER2) on their cell surface [1]. Identification of receptor expression offers treatment options for individualized targeted therapies.

\section{Human Epidermal Growth Factor Receptor 2}

HER2 is part of the ErbB family, which consists of 4 plasma membrane-bound receptor tyrosine kinases with HER1 (human epithelial growth factor receptor ErbB1), HER2 (Neu, ErbB2), HER3 (ErbB3), and HER4 (ErbB4). Signaling occurs through ligand-dependent activation by external ligands like epidermal growth factor (EGF) and transforming growth factor a (TGFa) or ligand-independent activation by receptor dimerization through interaction with other HER family members (homo-/heterodimerization). Specifically, in HER2, activation of signal transduction can also occur by proteolytic cleavage of the extracellular domain. Like other ErbB receptors, the HER2 receptor has an extracellular ligand binding domain, a transmembrane domain, and an intracellular domain which mediates cell proliferation and apoptosis. Through intrinsic intracellular protein tyrosine kinase activity, receptor dimerization causes autophosphorylation within the cytoplasmic domain and thereby downstream signaling via pathways like the mitogen-activated protein kinase (MAP kinase) and phosphatidylinositol 3-kinase (PI3K)/Akt pathways [2-5]. Overexpression of the proto-oncogene HER2 plays an important role in breast cancer and is also found in several other cancer types like stomach and ovarian cancer or endometrial carcinoma. Besides HER2 overexpression, other gene amplifications and alterations with spontaneous receptor dimerization in the absence of a ligand and receptor activation, with subsequent cell proliferation, have been described as well.

\section{KARGER \\ Fax +497614520714

\section{(c) 2015 S. Karger GmbH, Freiburg}

1661-3791/15/0103-0173\$39.50/0
Dr. Amelie Schramm

Department of Gynecology and Obstetrics University Hospital Ulm

Prittwitzstraße 43, 89075 Ulm, Germany

Amelie.Schramm@uniklinik-ulm.de 


\section{HER2 Definition}

Overexpression of HER2 in breast cancer is analyzed by immunohistochemistry (IHC) or fluorescent in situ hybridization (FISH) on histopathological samples of primary cancer or metastatic tissue.

According to the American Society of Clinical Oncology (ASCO)/College of American Pathologists (CAP), HER2 positivity is defined by an IHC score of $3 \pm$, with strong staining of more than $10 \%$ of all invasive tumor cells. An IHC score of $2+$ needs further investigation whereas IHC scores of 0 and $1+$ are representative of HER2 negativity.

For an additional determination after an IHC score of $2+$ and also as a standalone test, a FISH analysis can be performed. FISH is based on the HER2 gene copy number, i.e. the ratio between the HER2 gene copy number and the number of chromosome 17 centromeres (CEP17). The ASCO and CAP define the presence of a HER2 amplification in FISH analysis as a dual-probe HER2/CEP17 ratio of $\geq 2.0$ or a single-probe average HER2 copy number of $\geq$ 6.0 signals/cell [6].

\section{HER2-Targeted Drugs}

\section{Lapatinib}

Lapatinib is a dual tyrosine kinase inhibitor that binds to the intracellular ATP-binding pocket of the protein kinase domain of HER2. By binding, lapatinib prevents autophosphorylation of the cytoplasmic domain and thereby downstream signaling and tumor cell growth [7-9].

\section{Trastuzumab}

Trastuzumab is a monoclonal antibody that binds to the extracellular subdomain IV of HER2. Various, partly not well understood, mechanisms lead to the inhibition of tumor cell proliferation and tumor angiogenesis. Besides the blockade of ligand-independent HER2 signaling with the prevention of homodimerization and signal transduction, trastuzumab is thought to cause apoptosis by antibody-dependent cell-mediated cytotoxicity (ADCC). As another effect, trastuzumab also prevents the proteolytic cleavage of the extracellular domain [10-12].

\section{Pertuzumab}

Pertuzumab is a recombinant, humanized monoclonal antibody that acts as a HER2 dimerization inhibitor by binding to the extracellular dimerization domain. Heterodimerization with other HER2 family members like HER3 is blocked. This prevents ligandinitiated intracellular signaling through 2 major signaling pathways, the MAP kinase and PI3K pathways, and thereby cell cycle arrest of cells with HER2 overexpression. Also ADCC seems to be induced by pertuzumab. The combination of trastuzumab and pertuzumab acts synergistically, with complementary inhibition of HER2 signaling by blocking ligand-dependent and -independent signaling as well as homo- and heterodimerization [13-16].

\begin{tabular}{|c|c|c|c|}
\hline \multicolumn{4}{|c|}{ Adjuvant Studies for Her2-tarzeted Therapies } \\
\hline $\mathrm{CHT}$ and surgery $+/$ - RT & $\begin{array}{l}\text { Observation } \\
\text { H for } 52 \mathrm{w} \\
\mathrm{H}=\text { or } 104 \mathrm{w}\end{array}$ & & \\
\hline NSABP B-31 & $\begin{array}{l}=A C \times 4 \longrightarrow P(4 \times q 3 w ; 12 \times 0 \\
=A C \times 4 \Rightarrow P(4 \times q 3 w ; 12 \times\end{array}$ & (w) & \\
\hline NCCT6 N9831 & $\begin{array}{l}A C \times 4 \rightarrow 2 \times 12(\mathrm{cw}) \\
\mathrm{AC} \times 4 \rightarrow P \times 12(\mathrm{w}) \\
\mathrm{AC} \times 4 \rightarrow 2 \times 12(\mathrm{Cw})+\mathrm{H}\end{array}$ & $\underset{(52 \times \text { qw })}{H} \mathrm{H}\langle 52 \times$ qV & \\
\hline BCIRG OC6 & $\begin{array}{l}A C \times 4 \rightarrow D \times 4(q 3 w) \\
\rightarrow A C \times 4 \quad D \times 4(q 3 w)+1 \\
D+C a r b o(q 3 w \times 6)+H(18 \times\end{array}$ & $\begin{array}{ll}(12 \times q q u) & \rightarrow H \\
q(w) & \rightarrow H\end{array}$ & $\begin{array}{l}13 \times q 3 v s) \\
11 \times q 3 w\rangle\end{array}$ \\
\hline sungery $+/-\mathrm{CHT}$ & $\begin{aligned} & H(52 w) \\
= & L(52 w) \\
= & H(12 w) \rightarrow \text { wash out } 6 w \\
& H+L(52 w)\end{aligned}$ & - $\quad L(34 w)$ & 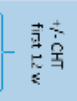 \\
\hline Tolaney et. Al & $\rightarrow P \times 12(q w)+H(12 w)$ & $H\langle 40 w\rangle$ & \\
\hline
\end{tabular}

Fig. 1. Summary of major adjuvant trials. $A=$ Doxorubicin, $C=$ cyclophosphamide, $\mathrm{CHT}=$ chemotherapy, $\mathrm{D}=$ docetaxel, $\mathrm{H}=$ trastuzumab, $\mathrm{L}=$ lapatinib, $\mathrm{P}=$ paclitaxel, $\mathrm{RT}=$ radiotherapy, $\mathrm{qw}=$ weekly administration, $\mathrm{q} 3 \mathrm{w}=$ every 3 weeks, $\mathrm{w}=$ week.

\section{Trastuzumab emtansine}

Trastuzumab emtansine (T-DM1) is the newest treatment option in HER2-positive breast cancer and unifies the idea of chemotherapy (CHT) and targeted therapies. In this drug, the cytotoxic and antimitotic maytansine derivative DM1 is linked to trastuzumab. Thereby, the CHT agent only acts in cells that present the targeted antigen on their cell surface, and systemic toxicity is limited. A current hypothesis suggests that the antitumor activity works through receptor-mediated internalization and intracellular release of DM1. By inhibition of microtubules, DM1 mediates its antiproliferative effects $[17,18]$.

\section{Combination of HER2-Targeted Therapies}

Up to $70 \%$ of all patients treated with trastuzumab-based therapy in the metastatic setting develop resistance against their prior HER2-targeted therapy [19-21]. To overcome this resistance, combined HER2-targeted therapies are tested in different variations. The combination of trastuzumab and pertuzumab effectively blocks tumor cell proliferation ex vivo and in vivo $[13,22]$. In the metastatic setting, dual antibody blockade has already been approved and is used in the clinical routine. Recent data show promising results also for the neoadjuvant setting (see 'Anti-HER2 in the Neoadjuvant Setting'). Also a combination of trastuzumab and lapatinib improves the clinical benefit rate (CBR) and progression-free survival (PFS) compared to lapatinib alone, in patients with metastatic breast cancer (MBC) progressing on prior trastuzumab treatment.

\section{HER2-Targeted Therapy in Clinical Trials}

\section{Adjuvant HER2 Treatment}

There were 4 important studies, first presented at the ASCO Annual Meeting 2005, which initiated trastuzumab use in adjuvant treatment (fig. 1). The Herceptin Adjuvant Trial (HERA NCT00045032) enrolled 5,090 women with HER2-positive early breast cancer after completion of locoregional therapy (surgery \pm radiotherapy) and at least 4 courses of CHT. Patients were randomized to either a control group or 1 or 2 years of treatment with 
trastuzumab. A first analysis (after 1 year (median) of follow-up) indicated the benefit of trastuzumab in adjuvant systemic therapy with an unadjusted hazard ratio (HR) of 0.54 for an event in the trastuzumab group (1 year) compared to the observation group [23]. A final comparison of the interventional arms showed no evidence of better outcome with 2 years versus 1 year of trastuzumab. Nevertheless, the number of adverse cardiac events slightly rises through 2 years of drug administration compared to 2 year. The HR for disease-free survival (DFS) and also for overall survival (OS) was 0.76 each, for 1 year of trastuzumab treatment versus observation, which confirms the efficacy of trastuzumab therapy [24].

The results of the National Surgical Adjuvant Breast and Bowel Project (NSABP) B-31 trial and the North Central Cancer Treatment Group (NCCTG) N9831 trial were presented in 1 analysis. In both trials, 4,046 women with HER2-positive early-stage breast cancer were treated with CHT comprising 4 cycles of doxorubicin and cyclophosphamide (AC) followed by paclitaxel for 3 months (weekly or q21d). The NSABP B-31 study randomized patients to CHT with or without 52 weeks of trastuzumab administration afterwards. The NCCTG N9831 study had 3 arms with only CHT, CHT plus trastuzumab simultaneously (with paclitaxel) or plus trastuzumab sequentially (after paclitaxel). The interim and final analyses of the trials did demonstrate the benefit of addition of trastuzumab for DFS and OS, by increasing the 10-year OS rate from $75.2 \%$ to $84 \%$, thus leading to a $37 \%$ relative improvement in OS (HR 0.63, P < 0.001). Also, the DFS benefits from trastuzumab, with an increase of the 10 -year DFS rate from $62.2 \%$ to $73.7 \%$. Thus, HER2 blockade results in a $40 \%$ (HR 0.60, P < 0.001) improvement of the DFS $[25,26]$. Further analysis in the NCCTG N9831 study was done regarding the efficacy of concurrent versus sequential administration of trastuzumab. Although the analysis results were not statistically significant, they showed a trend of a prolonged DFS in the concurrent treatment arm (concurrent/sequential HR 0.77; 99.9\% confidence interval (CI), 0.53-1.11, P = 0.02 ) and supported the recommendation of a concurrent regimen with taxane CHT [27].

Another adjuvant trial was the Breast Cancer International Research Group (BCIRG)-006 study (NCT00021255), which investigated the efficacy of trastuzumab as well as the effectiveness and safety of an anthracycline-free CHT regimen in order to reduce cardiac toxicity. After randomization, 3,222 women with HER2-positive early-stage high-risk breast cancer were treated with doxorubicin, cyclophosphamide, and docetaxel (ACT), ACT plus trastuzumab for 1 year $(\mathrm{AC}-\mathrm{TH})$, or with the non-anthracycline regimen consisting of docetaxel, carboplatin, and 52 weeks of trastuzumab (TCH). The final analysis underlined the importance of trastuzumab treatment, with superiority of both the trastuzumab-containing regimes compared to ACT (OS 87\%, DFS 75\%). Even though no significant difference in OS and DFS was seen for AC-TH (OS 92\%, DFS $84 \%$ ) and TCH (OS 91\%, DFS 81\%), there was a trend for better outcome in the AC-TH arm compared to TCH (124 vs. 144 distant relapse events). Cardiotoxicity was lower in the TCH arm. Especially for patients with high risk of congestive heart failure, the TCH regimen is thus an appropriate alternative [28].

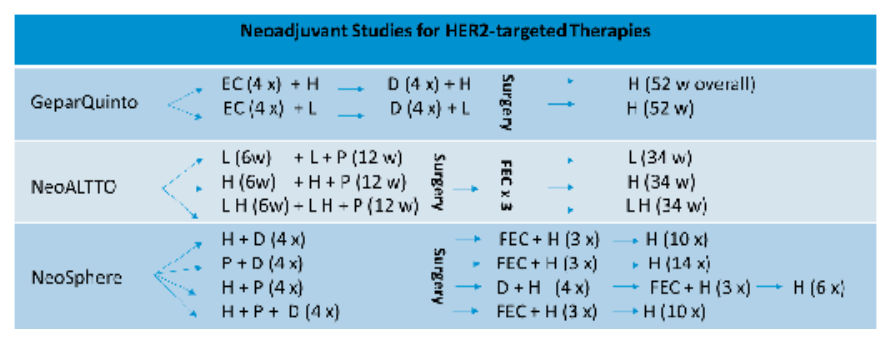

Fig. 2. Summary of major neoadjuvant trials. $\mathrm{E}=$ Epirubicin, $\mathrm{C}=$ cyclophosphamide, $\mathrm{D}=$ docetaxel, $\mathrm{FEC}=5$-fluorouracil, epirubicin and cyclophosphamide, $\mathrm{H}=$ trastuzumab, $\mathrm{L}=$ lapatinib, $\mathrm{w}=$ weeks.

The Adjuvant Lapatinib and/or Trastuzumab Treatment Optimisation (ALTTO - NCT00490139) trial was initiated to analyze the efficacy of combination therapy in adjuvant treatment. With more than 8,000 participants, the trial was the largest study on adjuvant therapy in HER2-positive breast cancer. Women were treated with surgery and CHT (anthracycline based vs. anthracycline free), followed by randomization to trastuzumab alone, lapatinib alone, trastuzumab followed by lapatinib, or concurrent therapy with lapatinib and trastuzumab. Anti-HER2 therapy was given for 52 weeks and was combined with paclitaxel/docetaxel in women with prior anthracycline-based CHT for the first 12 weeks. The lapatinib arm was prematurely closed since an interim analysis in 2011 suggested inferiority of this arm. First results were presented at the ASCO Annual Meeting 2014. After 4 years of followup, the DFS rate for trastuzumab plus lapatinib amounts to $88 \%$ compared to $86 \%$ for trastuzumab monotherapy (HR 0.84, 97.5\% CI $0.7-1.02, \mathrm{P}=0.048$ ) and $87 \%$ for trastuzumab followed by lapatinib versus $86 \%$ for trastuzumab monotherapy (HR 0.96, 97.5\% CI $0.8-1.25, \mathrm{P}=0.610$ ). In addition the combination of trastuzumab and lapatinib showed no benefit regarding OS compared to trastuzumab. Adverse events were more likely in patients within the lapatinib arm; the overall cardiac toxicity was tolerable [29].

For an evaluation of single standard treatment in patients with small, node-negative HER2-positive breast cancer, Tolaney et al. [30] recruited 406 patients in a single-group study. Women were treated with paclitaxel and trastuzumab for 12 weeks, followed by trastuzumab monotherapy for 40 weeks. The 3-year rate of survival free from invasive disease was $98.7 \%$ (95\% CI 97.6-99.8) and the 3 -year rate of recurrence-free survival was $99.2 \%$ (95\% CI $98.4-$ 100 ). With a risk of early recurrence of about $2 \%$ and a low rate of serous toxic effects (symptomatic congestive heart failure $0.5 \%$ ), treatment with adjuvant paclitaxel and trastuzumab could be considered as an option for node-negative, small HER2-postive breast cancer.

\section{Anti-HER2 in the Neoadjuvant Setting}

Neoadjuvant CHT offers an in vivo assessment of tumor response. One of the first trials (fig. 2) underlining the concept of dual blockade was the phase III GeparQuinto trial (NCT00567554), in which the addition of trastuzumab versus lapatinib to anthracycline-taxane-based neoadjuvant $\mathrm{CHT}$ was tested. 620 women with operable or locally advanced HER2-positive breast cancer were 
treated either with epirubicin, cyclophosphamide and docetaxel plus trastuzumab or the same CHT plus lapatinib for a total of 6 months and underwent surgery afterwards. The trastuzumab arm showed a significantly better pathological complete response (pCR) rate, with $30.3 \%$ versus $22.7 \%$ in the lapatinib group. Thus, the authors oppose the routine use of lapatinib as a single targeted agent in combination with neoadjuvant CHT [31].

In the NeoALTTO trial (NCT00553358), the combination of lapatinib and trastuzumab with neoadjuvant $\mathrm{CHT}$ was evaluated systematically. Women with operable HER2-positive breast cancer were assigned to treatment with paclitaxel and lapatinib only, trastuzumab only, or a combination of lapatinib and trastuzumab for 3 months. For all patients, paclitaxel CHT started only after 6 weeks of HER2 blockade. Subsequent to surgery, a systemic therapy with 5-fluorouracil, epirubicin, and cyclophosphamide (FEC) as well as completion of anti-HER2 therapy were performed (overall 52 weeks of lapatinib, trastuzumab, or lapatinib plus trastuzumab). No significant differences in the pCR rates were found for trastuzumab alone $(29.5 \%)$ and lapatinib alone $(24.7 \%)$ whereas the combination therapy of trastuzumab and lapatinib resulted in $\mathrm{pCR}$ rates of 51.3\%. Interestingly, women with HR-negative tumors had better pCR rates after combination therapy than patients with HRpositive tumors (61.33\% vs. $41.56 \%$; $95 \%$ CI). Differences were also seen in the lapatinib cohort (pCR rates: HR negative $33.78 \%$ vs. HR positive $16.25 \%$; $95 \% \mathrm{CI}$ ) and the trastuzumab group (36.49\% vs. $22.67 \%$; 95\% CI) [32].

Within the open-label phase II trial 'Neoadjuvant Study of Pertuzumab and Herceptin in an Early Regimen Evaluation' (NeoSphere - NCT00545688), 417 patients with locally advanced, inflammatory or early-stage invasive HER2-positive breast cancer were randomized into 4 therapy arms. Cohort A was treated with trastuzumab plus docetaxel, cohort B with pertuzumab, trastuzumab, and docetaxel, group $C$ received pertuzumab and trastuzumab, and group D received pertuzumab plus docetaxel for 4 neoadjuvant cycles. CHT was followed by surgery and adjuvant systemic therapy (further $\mathrm{CHT}$ and 52 weeks of trastuzumab). pCR and safety were evaluated, with significantly improved $\mathrm{PCR}$ rates found in group B with pertuzumab and trastuzumab plus docetaxel (45.8\%) compared to only docetaxel plus trastuzumab (group A; $29.0 \%$ ). Interestingly, group C had pCR rates of $16.8 \%$ with only dual HER2-targeted therapy; group D (pertuzumab plus docetaxel) had pCR rates of $24.0 \%$. Also in the NeoSphere trial, women with HR-negative tumors had better pCR rates than women with HRpositive tumors. The pCR rates for group A were 20.0\% (HR positive) versus $36.8 \%$ (HR negative), for group B $26.0 \%$ versus $63.2 \%$, for group C 5.9\% versus $27.3 \%$, and in group D $17.4 \%$ versus $30.0 \%$. Combination of trastuzumab and pertuzumab was not shown to increase cardiac toxicity [33].

\section{HER2 Blockade in MBC}

Several large trials have been conducted comparing single-agent CHT with or without trastuzumab (fig. 3), one of the first being the study by Slamon et al. [34], which started recruiting in 1995. 469 women were randomized to standard CHT with or without addi-

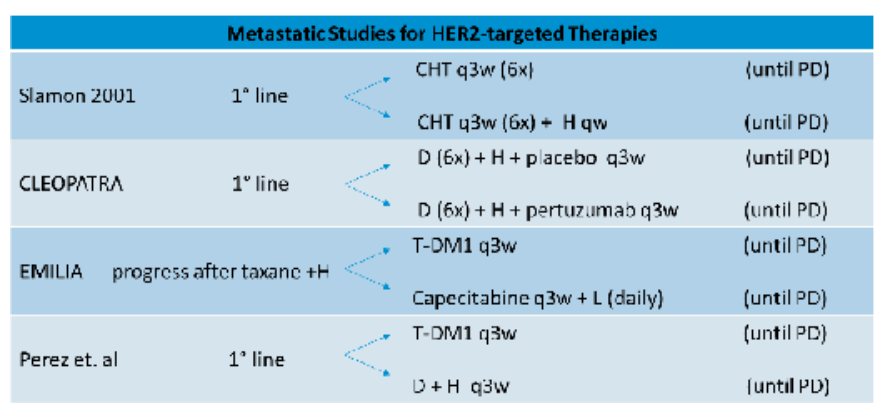

Fig. 3. Summary of major metastatic trials. $\mathrm{CHT}=$ Chemotherapy, $\mathrm{D}=$ docetaxel, $\mathrm{H}=$ trastuzumab, $\mathrm{L}=$ lapatinib, $\mathrm{PD}=$ progressive disease, $\mathrm{qw}=$ weekly administration, $\mathrm{q} 3 \mathrm{w}=$ every 3 weeks, $\mathrm{T}-\mathrm{DM} 1=$ trastuzumab emtansine.

tional trastuzumab $(\mathrm{H})$ administration. Anthracycline (A; either doxorubicin or epirubicin) plus cyclophosphamide (C) or paclitaxel $(\mathrm{P})$ were given for at least 6 courses; trastuzumab application did continue until disease progression. The results showed significantly increased clinical benefit for the combination of trastuzumab with first-line CHT. With trastuzumab, the time to disease progression was extended from 4.6 to 7.4 months (median) $(\mathrm{P}<$ 0.001 ), the rate of objective response (OR) was $50 \%$ compared to $32 \%(\mathrm{P}<0.001)$, and the median survival was prolonged to 25.1 compared to 20.3 months $(\mathrm{P}=0.01)$, even though the majority of patients without trastuzumab crossed over to trastuzumab in the second line. The risk of death was reduced by $20 \%$ with addition of trastuzumab. However, the risk for cardiac toxicity with cardiac dysfunction of New York Heart Association class III or IV was clearly higher in the trastuzumab groups, especially for women treated with trastuzumab and anthracycline-based $\mathrm{CHT}$ regimens (27\% in the $\mathrm{AC}+\mathrm{H}$ group; $8 \%$ in the group with $\mathrm{AC}$ alone; $13 \%$ in the $\mathrm{P}+\mathrm{H}$ group; and only $1 \%$ for $\mathrm{P}$ alone).

Several years later, the placebo-controlled CLEOPATRA trial (NCT00567190) analyzed the benefit of the addition of pertuzumab to therapy with docetaxel and trastuzumab in women with HER2-positive first-line MBC. With an improvement of 6.3 months (12.4 vs. 18.7 months), the PFS was significantly longer with pertuzumab/trastuzumab (HR 0.68, 95\% CI 0.58-0.80). Also the median OS was 15.7 months longer for patients randomized to the dual HER2-targeted therapy arm, with 56.5 versus 40.8 months for placebo plus trastuzumab [35].

The efficacy of TDM-1 in MBC was already proven by a couple of trials, e.g. the EMILIA trial (NCT00829166), in which 991 women with HER2-positive advanced breast cancer and previous treatment with trastuzumab plus taxane were randomly assigned to 2 treatment arms, one arm receiving T-DM1 and the other arm receiving lapatinib plus capecitabine. Whereas the PFS and median OS were significantly better for the T-DM1 arm (PFS: 9.6 vs. 6.4 months, HR 0.65, 95\% CI 0.55-0.77, P < 0.001; OS: 30.9 months vs. 25.1 months, HR $0.68,95 \%$ CI $0.55-0.85, \mathrm{P}<0.001$ ), the rate of toxicities was higher in the lapatinib/capecitabine cohort. About $57 \%$ of all patients suffered from adverse events grade 3 or 4 compared to only $41 \%$ in the TDM-1 group. According to these results, T-DM1 seems to be a great option in second-line therapy and is 
considered as first choice in that situation [18]. Another study, by Hurvitz et al. [36], analyzed the use of T-DM1 as first-line treatment in the metastatic setting with randomization of 137 patients to trastuzumab plus docetaxel or T-DM1. The PFS was significantly different, with 14.2 months in the T-DM1 group versus 9.7 months with trastuzumab/docetaxel. Also adverse events (grade 3 or higher) were less likely in the T-DM1 cohort (46.4\% vs. $90.9 \%$ ), thus supporting the positive safety profile of T-DM1.

\section{Summary}

Systemic treatment recommendations in HER2-positive breast cancer are highly influenced by clinical research and results from the described trials. The German 'Working Group for Gynecological Oncology' (AGO) recommends anti-HER2 treatment with trastuzumab for 1 year in addition to CHT as standard therapy for early HER2-positive breast cancer. Trastuzumab is recommended to be given concurrently to taxanes. For HER2-positive metastatic disease, CHT with docetaxel plus trastuzumab and pertuzumab is preferred. For progressive disease, TDM-1 and later combination of lapatinib and capecitabine are recommended [37, 38].

\section{Perspective}

Further trials try to investigate possible optimization of the targeted therapies in HER2-positive breast cancer may change the clinical routine in the treatment of HER2-positve breast cancer in the near future. Especially the efficacy of trastuzumab and pertuzumab for adjuvant (Aphinity study) and neoadjuvant treatment is of current research interest. T-DM1, at the moment recommended for second-line therapy in $\mathrm{MBC}$, is tested in several trials also for the (neo)adjuvant setting.

A further research interest is the investigation of the role of CHT in the presence of potent anti-HER2 blockade in patients with MBC. The Pernetta trial is a randomized phase II trial with 2 treatment arms. All patients with HER2-positive, first-line MBC are treated with dual HER2-targeted therapy (trastuzumab and pertuzumab). Cohort A only receives HER2 blockade whereas cohort $\mathrm{B}$ receives HER2 blockade with paclitaxel or vinorelbine (CHT regimen left to the discretion of the investigator). Patients with estrogen receptor (ER)-positive disease will get endocrine therapy in addition to antibody therapy (upfront in cohort A and after stop of $\mathrm{CHT}$ in cohort B). In case of disease progression, second-line CHT follows for all cohorts with T-DM1. The efficacy of dual HER2 inhibition is evaluated by OS at 24 months for the CHT-free and CHT-containing cohorts.

DETECT $\mathrm{V}$ is a randomized phase III study including women with HR-positive and HER2-positive MBC. Therein, the combination of a dual HER2-targeted therapy (pertuzumab and trastuzumab) with standard CHT is compared to a dual HER2-targeted therapy with endocrine therapy in several treatment lines. The safety and tolerability of the dual HER2 therapy are analyzed by the determination of adverse events.

\section{Conclusions}

Targeted therapies offer selective treatment options; in case of HER2-positive breast cancer, they emerged as real game changers with most often very little additional toxicity. Especially in HER2positive breast cancer, targeted therapies are well established and have been proven as irreplaceable part of anticancer strategies. New substances and combinations are expected to enter the clinical routine in the near future. HER2-targeted therapies are excellent examples of the effective advancement of molecular findings to clinical use by translational research and by using individual characteristics of malignant tumors.

\section{Disclosure Statement}

J.H. is a member of the Roche, GSK, and Novartis advisory boards. All other authors do not have any conflicts of interest.

\section{References}

1 Mitri Z, Constantine T, O'Regan R: The HER2 receptor in breast cancer: pathophysiology, clinical use, and new advances in therapy. Chemother Res Pract 2012; 2012:743193.

2 Graus-Porta D, Beerli RR, Daly JM, Hynes NE: ErbB-2, the preferred heterodimerization partner of all ErbB receptors, is a mediator of lateral signaling. EMBO J 1997;16:1647-1655.

3 Hynes NE, Stern DF: The biology of erbB-2/neu/ HER-2 and its role in cancer. Biochim Biophys Acta 1994;1198:165-184.

4 Ménard S, Tagliabue E, Campiglio M, Pupa SM: Role of HER2 gene overexpression in breast carcinoma. J Cell Physiol 2000;182:150-162.

5 Olayioye MA: Update on HER-2 as a target for cancer therapy: intracellular signaling pathways of ErbB2/ HER-2 and family members. Breast Cancer Res 2001;3: 385-389.
6 Wolff AC, Hammond MEH, Hicks DG, Dowsett M, McShane LM, Allison KH, et al.: Recommendations for human epidermal growth factor receptor 2 testing in breast cancer: American Society of Clinical Oncology/ College of American Pathologists clinical practice guideline update. Arch Pathol Lab Med 2014;138:241-256.

7 Konecny GE, Pegram MD, Venkatesan N, Finn R, Yang G, Rahmeh M, et al.: Activity of the dual kinase inhibitor lapatinib (GW572016) against HER-2-overexpressing and trastuzumab-treated breast cancer cells. Cancer Res 2006;66:1630-1639.

8 Spector NL, Xia W, Burris H, Hurwitz H, Dees EC, Dowlati A, et al.: Study of the biologic effects of lapatinib, a reversible inhibitor of ErbB1 and ErbB2 tyrosine kinases, on tumor growth and survival pathways in patients with advanced malignancies. J Clin Oncol 2005;23:2502-2512.
9 Rusnak DW, Lackey K, Affleck K, Wood ER, Alligood KJ, Rhodes N, et al.: The effects of the novel, reversible epidermal growth factor receptor/ErbB-2 tyrosine kinase inhibitor, GW2016, on the growth of human normal and tumor-derived cell lines in vitro and in vivo. Mol Cancer Ther 2001;1:85-94.

10 Valabrega G, Montemurro F, Aglietta M: Trastuzumab: mechanism of action, resistance and future perspectives in HER2-overexpressing breast cancer. Ann Oncol 2007;18:977-984.

11 Hudis CA: Trastuzumab - mechanism of action and use in clinical practice. N Engl J Med 2007;357:39-51.

12 Spector NL, Blackwell KL: Understanding the mechanisms behind trastuzumab therapy for human epidermal growth factor receptor 2-positive breast cancer. J Clin Oncol 2009;27:5838-5847. 
13 Nahta R, Hung M-C, Esteva FJ: The HER-2-targeting antibodies trastuzumab and pertuzumab synergistically inhibit the survival of breast cancer cells. Cancer Res 2004;64:2343-2346.

14 Agus DB, Gordon MS, Taylor C, Natale RB, Karlan B, Mendelson DS, et al.: Phase I clinical study of pertuzumab, a novel HER dimerization inhibitor, in patients with advanced cancer. J Clin Oncol 2005;23:2534-2543.

15 Baselga J, Gelmon KA, Verma S, Wardley A, Conte P, Miles D, et al.: Phase II trial of pertuzumab and trastuzumab in patients with human epidermal growth factor receptor 2-positive metastatic breast cancer that progressed during prior trastuzumab therapy. J Clin Oncol 2010;28:1138-1144

16 Lynce F, Swain SM: Pertuzumab for the treatment of breast cancer. Cancer Invest 2014;32:430-438.

17 Lewis Phillips GD, Li G, Dugger DL, Crocker LM, Parsons KL, Mai E, et al.: Targeting HER2-positive breast cancer with trastuzumab-DM1, an antibody-cytotoxic drug conjugate. Cancer Res 2008;68:9280-9290.

18 Verma S, Miles D, Gianni L, Krop IE, Welslau M, Baselga J, et al.: Trastuzumab emtansine for HER2-positive advanced breast cancer. N Engl J Med 2012;367: 1783-1791.

19 Wong DJL, Hurvitz SA: Recent advances in the development of anti-HER2 antibodies and antibody-drug conjugates. Ann Transl Med 2014;2:122.

20 Brufsky AM: Current approaches and emerging directions in HER2-resistant breast cancer. Breast Cancer (Auckl) 2014;8:109-118.

21 Valabrega G, Montemurro F, Sarotto I, Petrelli A, Rubini $\mathrm{P}$, Tacchetti C, et al.: TGFalpha expression impairs trastuzumab-induced HER2 downregulation. Oncogene 2005;24:3002-3010.

22 Scheuer W, Friess T, Burtscher H, Bossenmaier B, Endl J, Hasmann M: Strongly enhanced antitumor activity of trastuzumab and pertuzumab combination treatment on HER2-positive human xenograft tumor models. Cancer Res 2009;69:9330-9336.
23 Piccart-Gebhart MJ, Procter M, Leyland-Jones B, Goldhirsch A, Untch M, Smith I, et al.: Trastuzumab after adjuvant chemotherapy in HER2-positive breast cancer. N Engl J Med 2005;353:1659-1672.

24 Goldhirsch A, Gelber RD, Piccart-Gebhart MJ, de Azambuja E, Procter M, Suter TM, et al.: 2 years versus 1 year of adjuvant trastuzumab for HER2-positive breast cancer (HERA): an open-label, randomised controlled trial. Lancet 2013;382:1021-1028.

25 Perez EA, Romond EH, Suman VJ, Jeong J-H, Davidson NE, Geyer CE, et al.: Four-year follow-up of trastuzumab plus adjuvant chemotherapy for operable human epidermal growth factor receptor 2-positive breast cancer: joint analysis of data from NCCTG N9831 and NSABP B-31. J Clin Oncol 2011;29:33663373.

26 Perez EA, Romond EH, Suman VJ, Jeong J-H, Sledge G, Geyer CE, et al.: Trastuzumab plus adjuvant chemotherapy for human epidermal growth factor receptor 2-positive breast cancer: planned joint analysis of overall survival from NSABP B-31 and NCCTG N9831. J Clin Oncol 2014;32:3744-3752.

27 Perez EA, Suman VJ, Davidson NE, Gralow JR, Kaufman PA, Visscher DW, et al.: Sequential versus concurrent trastuzumab in adjuvant chemotherapy for breast cancer. J Clin Oncol 2011;29:4491-4497.

28 Slamon D, Eiermann W, Robert N, Pienkowski T, Martin M, Press M, et al.: Adjuvant trastuzumab in HER2-positive breast cancer. N Engl J Med 2011;365: 1273-1283.

29 Crozier JA, Perez EA: Perspectives from the American Society of Clinical Oncology 2014 Conference: breast cancer highlights. Future Oncol 2014;10:1897-1899.

30 Tolaney SM, Barry WT, Dang CT, Yardley DA, Moy B, Marcom PK, et al.: Adjuvant paclitaxel and trastuzumab for node-negative, HER2-positive breast cancer. N Engl J Med 2015;372:134-141.
31 Untch M, Loibl S, Bischoff J, Eidtmann H, Kaufmann $\mathrm{M}$, Blohmer J-U, et al.: Lapatinib versus trastuzumab in combination with neoadjuvant anthracycline-taxanebased chemotherapy (GeparQuinto, GBG 44): a randomised phase 3 trial. Lancet Oncol 2012;13:135-144.

32 Baselga J, Bradbury I, Eidtmann H, Di Cosimo S, de Azambuja E, Aura C, et al.: Lapatinib with trastuzumab for HER2-positive early breast cancer (NeoALTTO): a randomised, open-label, multicentre, phase 3 trial. Lancet 2012;379:633-640.

33 Gianni L, Pienkowski T, Im Y-H, Roman L, Tseng L-M, Liu M-C, et al.: Efficacy and safety of neoadjuvant pertuzumab and trastuzumab in women with locally advanced, inflammatory, or early HER2-positive breast cancer (NeoSphere): a randomised multicentre, open-label, phase 2 trial. Lancet Oncol 2012;13:25-32.

34 Slamon DJ, Leyland-Jones B, Shak S, Fuchs H, Paton $\mathrm{V}$, Bajamonde $\mathrm{A}$, et al.: Use of chemotherapy plus a monoclonal antibody against HER2 for metastatic breast cancer that overexpresses HER2. N Engl J Med 2001;344:783-792.

35 Swain SM, Baselga J, Kim S-B, Ro J, Semiglazov V, Campone $\mathrm{M}$, et al.: Pertuzumab, trastuzumab, and docetaxel in HER2-positive metastatic breast cancer. N Engl J Med 2015;372:724-734.

36 Hurvitz SA, Dirix L, Kocsis J, Bianchi G V, Lu J, Vinholes J, et al.: Phase II randomized study of trastuzumab emtansine versus trastuzumab plus docetaxel in patients with human epidermal growth factor receptor 2-positive metastatic breast cancer. J Clin Oncol 2013; 31:1157-1163

37 Liedtke C, Thill M, Hanf V, Schütz F: AGO recommendations for the diagnosis and treatment of patients with early breast cancer: update 2014. Breast Care (Basel) 2014;9:189-200.

38 Hanf V, Schütz F, Liedtke C, Thill M: AGO recommendations for the diagnosis and treatment of patients with advanced and metastatic breast cancer: update 2014. Breast Care (Basel) 2014;9:202-209. 